\title{
Qui est vulnérable ? Une analyse critique du discours public de l'agence européenne Frontex
}

Who Is Vulnerable? A Critical Analysis of the Public Narrative of the European Agency Frontex

¿Quién es vulnerable? Un análisis crítico del discurso público de la agencia europea Frontex

\section{Cécile Dubernet}

\section{(2) OpenEdition}

\section{Journals}

Édition électronique

URL : https://journals.openedition.org/remi/11181

DOI : $10.4000 /$ remi. 11181

ISSN : $1777-5418$

Éditeur

Université de Poitiers

Édition imprimée

Date de publication : 1 octobre 2018

Pagination : 205-227

ISBN : 979-10-90426-62-7

ISSN : 0765-0752

\section{Référence électronique}

Cécile Dubernet, «Qui est vulnérable? Une analyse critique du discours public de l'agence européenne Frontex », Revue européenne des migrations internationales [En ligne], vol. 34 - n² et 3 | 2018, mis en ligne le 01 janvier 2021, consulté le 14 avril 2022. URL : http://journals.openedition.org/remi/11181 ; DOl : https://doi.org/10.4000/remi.11181 


\section{Qui est vulnérable? Une analyse critique du discours public de I'agence européenne Frontex}

\section{Cécile Dubernet ${ }^{1}$}

La "crise" migratoire qui secoue l'Europe donne lieu à de multiples éditoriaux et commentaires politiques ainsi qu'à des articles scientifiques débattant âprement de la raison d'être et du futur des accords de Schengen comme du régime de I'asile. Au cœur de la tourmente se trouve Frontex, qui se définit comme "l'agence pour la gestion de la coopération opérationnelle aux frontières extérieures des États membres de I'Union européenne". Créée en 2004 dans la vague sécuritaire qui a suivi les attentats du 11 septembre 2001, Frontex a progressivement pris une place prépondérante dans la formulation et la mise en œuvre de politiques anti-migratoires aux frontières de I'Union (Leonard, 2010; Campesi, 2014).

De nombreux travaux examinent l'efficacité de ces politiques d'étanchéité et leur ambiguïté vis-à-vis des droits humains fondamentaux (Fink, 2012; Horsti, 2012; Intran et Sibley, 2014; Ottavy et Clochard, 2014; Campesi, 2014; Peyronnet, 2015) ${ }^{2}$. Peu d'études néanmoins examinent les discours européens sur la frontière, si ce n'est pour en confronter quelques citations aux chiffres de la réalité, parfois en montrer les incohérences, en dénoncer l'hypocrisie (Horsti, 2012; Beauchemin et Lessault, 2014; Campesi, 2014). À ce titre, les études de Horsti et de Campesi sont des plus intéressantes en ce qu'elles discutent de l'émergence d'une rhétorique humanitaire dans la communication institutionnelle de Frontex. Les auteurs suggèrent que l'agence a su adopter un langage humanitaire et urgentiste pour se positionner comme acteur central des politiques d'asile, des opérations de recherche et sauvetage (SAR), en même temps qu'elle continue de promouvoir des approches militarisées de la migration.

Alors que Frontex s'est restructuré en 2016, les recherches pré-citées appellent une investigation plus approfondie et plus rigoureuse du discours institutionnel de l'agence. Cet article présente les résultats d'une étude discursive critique des

\footnotetext{
1 Enseignante-chercheuse, Institut catholique de Paris, EA Religion, culture et société, 21 Rue d'Assas, 75006Paris ; c.dubernet@icp.fr

2 Les réseaux de défense des droits des migrants, tels que Migreurop, et des associations telles la Cimade, Human Rights Watch, la FIDH entre autres produisent aussi régulièrement des études bien documentées sur le travail de Frontex. Sur cette thématique, chercheurs et militants se retrouvent dans des espaces partagés dont le réseau TERRA $\mathrm{HN}$ est une bonne illustration.
} 
positions publiques de l'organisation sur toute sa période opératoire (2006-2016). II s'agit d'offrir une revue systématique de l'usage du champ lexical anglais de la vulnérabilité par Frontex, d'en identifier les concepts dominants ainsi que leurs interactions. Dans la lignée de l'approche discursive critique de Horsti (2012), cette étude sémantique permet donc de tester I'hypocrisie dénoncée par les ONG, des journalistes et universitaires (Guiraudon, 2008; Rodier, 2010; Campbell, 2016) ainsi que la réalité d'une inflexion humanitaire du discours de l'agence à partir de 2010-2011 telle que repérée et décrite par Campesi (2014). Elle permet également de mieux appréhender le positionnement de l'agence vis-à-vis de ses partenaires et soulève les questions de la marginalisation de la protection juridique et de la performativité d'un discours institutionnel. Enfin, au-delà même de Frontex, l'article illustre, dans le champ migratoire, l'usage du concept de vulnérabilité très en vogue en sciences sociales. Dans la lignée des travaux de Bresson, Geronimi et Pottier ou encore Thomas (Bresson et al., 2013; Thomas, 2008 et 2010), et en complémentarité avec l'éditorial de Cambrezy et Charbit du numéro spécial de la REMI sur la vulnérabilité en 2007, il contribue à la construction d'un regard critique sur le sujet.

Le corpus analysé est constitué de l'intégralité des communiqués de presse de l'agence et des rapports associés en pièce jointe sur la période 2006-2016, soit environ 360 communiqués plus une soixantaine de rapports fournis (de quarante à quatre-vingt pages en moyenne). Une attention particulière a été accordée à l'iconographie des documents. La première partie de l'article, consacrée à la méthodologie, justifie le choix du corpus et la méthode d'investigation. La seconde présente les résultats de l'enquête. Enfin, la dernière confronte I'enquête à la littérature scientifique pour ouvrir sur plusieurs pistes de réflexion.

\section{Dix ans de projection institutionnelle passée sous crible}

Aborder une combinaison de trois sujets d'analyse aussi populaires que Frontex, la vulnérabilité et les questions migratoires est une entreprise risquée méthodologiquement. II est notamment impossible de prétendre à l'exhaustivité bibliographique. Pourtant, se positionner à la croisée des trois termes est envisageable dans la mesure où l'on établit une démarche ciblée, donc limitée. Les paragraphes ci-dessous clarifient les paramètres de la recherche : ils justifient le choix de l'objet d'enquête, expliquent la nature des archives étudiées, leur représentativité ainsi que l'approche linguistique choisie. Sont aussi abordées les limites de l'enquête.

\section{L'énonciateur : Frontex, produit de Schengen}

L'agence Frontex, contraction de Frontières Extérieures a été créée en $2004^{3}$ dans un contexte tendu, marqué par trois phénomènes : la mise en place de I'espace Schengen malgré des difficultés d'harmonisation des politiques migratoires des membres de l'UE, l'émergence de politiques internationales contre la traite humaine, enfin la "guerre contre le terrorisme" lancée en 2001. Agence

3 Council Regulation (EC) N²007/2004 du 26 octobre 2004, [en ligne], consulté le 27/08/2016. URL : http://frontex.europa.eu/assets/About_Frontex/frontex_regulation_en.pdf 
financée principalement par la Commission européenne, Frontex a vu son budget augmenter de façon spectaculaire pour atteindre plus de 250 millions d'euros en $2016^{4}$. Son mandat recouvre les opérations conjointes (dont les opérations de renvoi des migrants), la formation des gardes-frontières, l'analyse de risques, la recherche, les systèmes d'information et, depuis 2008, l'intervention rapide ${ }^{5}$.

Depuis sa création, Frontex se pose comme expert, venant en soutien des États membres dans cette fonction régalienne clé qu'est le contrôle aux frontières. En ce sens, l'agence se veut technique et refuse de porter la responsabilité politique des choix migratoires européens. Son refus de commenter l'accord passé en avril 2016 entre I'UE et la Turquie est une illustration récente de sa réticence à s'engager sur des terrains politiques controversés ${ }^{6}$. Son mandat reste donc centré sur l'étanchéité des frontières et la performance dans le contrôle des flux. Néanmoins, comme son nom le suggère, Frontex est au front. C'est l'organisme européen le plus en contact physique avec les migrants. Avec son approche technicienne, désincarnée, l'agence aborde les migrants comme $\mathrm{d}^{\prime}$ 'autres flux et des personnes sans documentation comme des flux illégaux. Ces voyageurs sont donc tracés, poursuivis, saisis, stockés (internés), parfois renvoyés. L'arsenal quasi militaire développé pour sécuriser les frontières européennes, en dépit d'un mandat qui fait référence à la charte européenne des droits fondamentaux, provoque la controverse. L'agence est sévèrement critiquée pour violations systématiques des droits des réfugiés, via ses politiques d'externalisation de l'asile qui préemptent la fuite (Fink, 2012; Ottavy et Clochard, 2014; Bauloz, 2017) et ses violations de l'article 33 de la Convention de 1951 pourtant ratifiée par tous les pays européens (Amnesty International, 2013; United Nations, 2013). Sous pression, Frontex a créé un Comité consultatif sur les droits fondamentaux ce qui n'a pas empêché l'émergence de campagnes civiques pour son démantèlement ${ }^{7}$.

À la croisée des contradictions européennes sur les questions migratoires, Frontex est donc un objet d'étude remarquable. Active dans un contexte corrosif, l'agence s'est aussi faite locutrice en développant un langage opératoire adressé à ses sponsors, partenaires, à la presse et au grand public. De controverses en crises, sa communication institutionnelle lui a permis de se défendre, de se cacher derrière un mandat, d'esquiver des accusations. En 2016, l'exemple le plus flagrant de cette gymnastique linguistique fut la décision de renommer l'Agence, sans pour autant changer l'orientation de sa mission. Frontex, chiffon rouge du monde associatif de défense des droits des migrants, devient alors I'European Border and Coast Guard Agency et travaillera en plus étroite coopé-

4 Frontex, documentation budgétaire, budget 2016, 24/12/2015, [en ligne], consulté le 24/07/2016. URL : http://frontex.europa.eu/assets/About_Frontex/Governance_documents/ Budget/Budget_2016.pdf

5 Synthétisé et traduit de l'onglet «Missions and Tasks», [en ligne], consulté le 29/04/16. URL : www.frontex.europe.eu

6 «Frequently Asked Questions» du site de l'agence précise qu'elle n'est qu'opérationnelle et ne peut être tenue responsable des décisions politiques des membres de I'Union européenne.

7 Les campagnes Frontexplode ou encore Frontexit dont les critiques acerbes tiennent bien dans ce slogan "démesuré, opaque, dangereux", [en ligne], consulté le 27/08/16. URL : www.frontexit.org 
ration encore avec les services douaniers des États membres, sur un budget largement renforcé jusqu'à $2020^{\circ}$.

\section{L'énoncé : le corpus dans son contexte}

Au fil des années, les communiqués de presse se sont multipliés constituant une archive vivante faite de plusieurs centaines de textes. À partir de 2010, ils sont souvent complétés par des rapports mis en lien en fin de texte. Ces derniers, plus longs, proposent des données souvent chiffrées sur une des missions premières de Frontex, I'analyse de risques, la veille technologique ou encore les opérations conjointes. Avec des titres fonctionnels, parfois accrocheurs ("Swedish officer injured in Lesbos " "Greece asks Frontex for rapid intervention teams in the Aegan Islands "), avec des structures grammaticales pré-formulées pour journalistes (notamment des références à Frontex à la troisième personne), avec des citations préfabriquées, le corpus fournit une excellente illustration de ce que Geert Jacobs nomme le discours projeté (Jacobs, 1998; Sleurs et al., 2003). Outre les journalistes, les nombreuses invitations à participer à des événements Frontex (conférences et présentations) suggèrent que les partenaires industriels et gouvernementaux sont des récipiendaires privilégiés, bien plus que les ONG (rarement mentionnées), le grand public ou les migrants eux-mêmes. II est d'ailleurs à noter que les versions non anglophones du site sont difficilement accessibles et restent incomplètes. Le corpus complet n'est accessible qu'en anglais ce qui limite grandement l'accès des publics non professionnels.

Bien que petit pour une analyse sémantique, le corpus choisi est valide méthodologiquement, car représentatif : il est de source primaire unique, complet et couvre toute la période opératoire de l'agence depuis les premières opérations conjointes Hera I et II en 2006. Par ailleurs, c'est aussi un corpus en expansion. Les crises ayant ponctué la production de ces archives doivent être prises en compte pour mesurer l'évolution du discours. Le nombre de communiqués a fortement augmenté entre 2006 (six communiqués) et 2016 (soixante-dix-neuf) au fil des crises, avec un record de quatre-vingt-huit communiqués de presse en 2015. Frontex communique en priorité sur ses activités : opérations conjointes, signatures d'accord avec des pays tiers et production de rapports d'analyse de risques $^{9}$. L'augmentation des matériaux mis en ligne annuellement n'est pas surprenante compte tenu de la croissance de l'organisation et de l'usage grandissant d'internet comme outil de projection dans la sphère publique. À titre de comparaison néanmoins, sur l'année 2015, le Haut Commissariat aux réfugiés de I'ONU (HCR) a produit près de 350 communiqués de presse. Sachant que le budget du HCR est d'environ 5 milliards de dollars soit environ vingt fois celui de Frontex, il est clair que ce dernier utilise l'outil du communiqué de presse proportionnellement plus que le HCR. De façon générale, le site de Frontex s'est imposé comme point d'entrée pour journalistes. Enfin, en dix ans, les communiqués de presse ne se sont pas seulement multipliés, mais aussi consolidés dans le sens où le texte passé devient contexte - ou texte connu - et fournit

8 Frontex, "Frontex welcomes European Parliament vote on European Border and Coast Guard", communiqué de presse, 06 juillet 2016.

9 Certains des rapports comportent des mentions indiquant qu'ils ont été validés "for public release". L'organisation distingue donc clairement ce qui peut être divulgué de ce qui doit rester à usage interne. 
donc un cadre mental de production et d'interprétation du discours (Van Dijk, 2009). On notera par exemple que les communiqués renvoient régulièrement à des rapports, et que ces derniers sont numérotés, donc s'additionnent et se complètent. À partir d'un document, il est également toujours possible de cliquer sur l'icône Frontex pour être renvoyé sur le site internet sur lequel, de nombreuses photos et des films institutionnels sur le travail des policiers accueillent le visiteur. Cette structure de l'information et l'interaction entre les différents textes ouvre la porte tant à des analyses intertextuelles qu'à des analyses de corpus. Dans la lignée de Baker et al. (2008), notre travail combine un cadrage critique et une analyse de corpus centrée sur les questions de vulnérabilité. L'étude n'aborde donc pas directement les questions d'intertextualité, mais offre une base et quelques pistes le permettant.

\section{Un cadrage critique pour décoder des silences et des absences}

Inspiré des théories sociales constitutives, I'analyse discursive critique aborde les données linguistiques comme des pratiques sociales reflétant et produisant de l'idéologie (Fairclough, 1995 et 2003). L'accent est mis sur les contextes, les questions de pouvoir, d'inégalité, de discrimination ou encore de changements décodés au cœur de discours institutionnels. Les approches critiques vont plus loin que l'analyse discursive classique en explorant non seulement le texte (collocations, métaphores et autres figures rhétoriques), mais aussi son contexte (mode de production, cible de consommation) et ses arrière-plans (Baker et al., 2008). Cette démarche permet de mettre à jour l'archéologie d'une organisation (Schneider, 2013). Dans notre cas, outre les remarques sur le contexte de l'énoncé déjà faites, il s'agit d'identifier des sujets et concepts dominants dans les positions publiques de Frontex, mais aussi d'autres qui manquent, des silences de l'agence. Le corpus étant complet, une telle ambition est possible et valide méthodologiquement. Par exemple, en scannant manuellement I'ensemble des communiqués, on note qu'il contient bien moins de références à la lutte contre la contrebande (drogue, produits de contrefaçon, etc.) qu'à la lutte contre l'immigration irrégulière alors que le mandat de l'agence recouvre tous les services de douane. Le terme racine "drug" n'apparaît qu'une trentaine de fois en dix ans, la plupart du temps dans des listes d'activités criminelles. Une demi-douzaine d'opérations de saisie sont mentionnées entre 2014 et 2016. De façon générale, alors que l'on trouve moins d'une dizaine de références à des produits illicites (drogues, biens, cigarettes, armes), le terme migration illégale ou irrégulière revient plus de quatre-vingt fois et le terme racine "migrant " près de 600 fois saturant les écrits. La priorité que Frontex projette est donc bien la

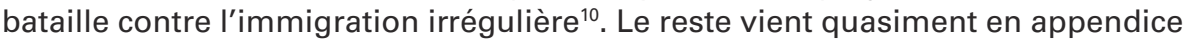
comme le souligne, de façon anodine, le communiqué de presse annonçant la refondation de l'agence :

10 Les termes «illegal» et "irregular» ont été tous les deux très utilisés par l'agence dans les communiqués de presse sur la période 2006-2016, le premier un peu plus que le deuxième. La différence n'est cependant pas très signifiante (192/154). Néanmoins, sachant que les ONG s'opposent à l'usage du terme "illegal " compte tenu de la criminalisation qu'il induit, il pourrait être intéressant, dans un autre travail, d'affiner la comparaison de l'usage des deux termes et de son évolution. 
" Many Frontex operations already have a multipurpose character, where in addition to detection of irregular migrants Frontex vessels and planes detect drug and weapon smuggling and track illegal fishing and reports oil spills. $n^{11}$

Même dans le champ sémantique de la migration, des orientations sont visibles : le corpus contient très peu d'information sur les opérations de renvoi de migrants. Par ailleurs, Frontex répond rarement et indirectement aux multiples accusations qui lui sont portées ${ }^{12}$. Le terme "Frontexit ${ }^{13}$ n'est jamais mentionné dans le corpus. De même, on ne trouve aucune mention d'organisations qui se sont opposées à son travail. De façon générale, les organisations de la société civile ne sont pas listées comme partenaires de l'agence. Ces absences donnent l'impression d'un discours en dialogue avec les mondes industriels et institutionnels uniquement, impression sur laquelle la troisième partie de I'article reviendra.

Enfin, les références au droit sont peu nombreuses et principalement inscrites dans des formules titres ("Convention Européenne des Droits Fondamentaux») ou si conventionnelles qu'elles sont peu signifiantes ${ }^{14}$. En dix ans on compte une seule mention explicite du principe de non-refoulement, et ce en mars 2016 dans le contexte de l'accord UE-Turquie pour la "ré-admission" de réfugiés syriens en Turquie.

"The readmission operations will proceed in full compliance with EU and international law, as well as the principle of non-refoulement. $»^{15}$

Ironiquement, on cite le principe alors même qu'on le bafoue. Par ailleurs, le terme "ré-admission " est une excellente illustration d'innovation linguistique destinée à confondre le lecteur en suggérant plus de contrôle qu'il n'y en a en réalité : les réfugiés syriens concernés n'ont été admis ni en Europe, ni même en Turquie. Ils ont simplement forcé les frontières. Parler de réadmission enTurquie postule l'existence de processus administratifs établis, cohérents et validés juridiquement qui n'existaient pourtant pas en mars 2016. De plus Frontex ne dit rien des promesses européennes de financement et de libéralisation de la politique de visa pour la Turquie, ni d'éventuelles procédures de demandes d'asile externalisées en Turquie. Le terme "ré-admission» est donc un euphémisme couvrant un accord décidé dans l'urgence, peu construit, violant le principe de non-refoulement et ajoutant à la confusion. Lire Frontex fait donc entrer dans un

11 Frontex, "5th Meeting of Contact Group of European Coastguard Functions hosted in Warsaw ", communiqué de presse, 26 mai 2016.

12 En 2007, une intervention défensive de son directeur minimise les responsabilités politiques de I'organisation. En 2011, alors qu'un rapport de Human Rights Watch accuse I'organisation de violer la Charte Européenne des Droits de I'Homme lors d'opérations menées en Grèce, Frontex réitère que l'organisation ne peut être tenue responsable du comportement des États membres.

13 Frontexit est une campagne lancée par un collectif de dix-neuf associations de défense des migrants pour informer les citoyens des activités de Frontex et en dénoncer les dérives, [en ligne], consulté le 10/09/17. URL : www.frontexit.org

14 Par exemple, "The process of returning people on joint return flights is governed by strict procedures to ensure full respect for fundamental rights and the safety of returnees and other participants ", communiqué de presse, 09 décembre 2013.

15 Frontex, "Frontex calls on Member States to deploy more officers to Greece", communiqué de presse, 23 mars 2016. 
monde d'expertise et de contrôle, un univers de jargon, de chiffres et d'action. $C^{\prime}$ est un espace dans lequel le professionnalisme hyper-technologique se pose, au-delà de toute critique, comme solution face à la criminalité transfrontalière. Impressionné par ce langage assuré, technocratique, par le martèlement de I'idée que Frontex maîtrise la bataille contre le crime, l'internaute pourrait même douter de la pertinence d'une enquête sur la vulnérabilité dans un tel corpus. Après tout, les forces de police s'inquiètent rarement du bien-être des criminels, et peu de celui des victimes. Seul le contexte d'urgence humanitaire dans lequel se meut Frontex, ses silences, et les accusations d'hypocrisie lancées régulièrement à l'encontre de l'agence nous incitent à pousser l'enquête.

\section{"En-quête " de vulnérabilité : choix des mots et dépouillement}

L'analyse discursive critique admet que tout est interprétation. Cette approche requiert non seulement d'expliciter la méthodologie, mais aussi d'avoir une grande transparence axiologique par laquelle le chercheur expose honnêtement les fondements de ce qu'il cherche et de ce qu'il croit. En l'occurrence, au cœur de notre enquête linguistique dans la fabrique du discours de Frontex se pose la question suivante : qui est vulnérable dans l'histoire contée par Frontex pendant dix ans?

Est vulnérable étymologiquement celui/celle qui est susceptible d'être blessé. Le terme vulnérabilité, lui, dénote une catégorie d'analyse reposant sur l'identification de groupes cibles, objets d'analyse, et d'un ou de plusieurs risques ainsi que de la probabilité de leur actualisation (Bresson et al., 2013). C'est un thème populaire depuis une trentaine d'années dans divers champs sociaux, y compris dans le champ des migrations (Cambrezy, 2007) ${ }^{16}$. Cependant, l'étude de la vulnérabilité reste source de controverse tant la recherche de cadres d'analyse captant le phénomène est, elle-même, source de contrôle et de pouvoir sur les personnes décrétées "vulnérables" (Thomas, 2008 et 2010), tant la production de telles catégories induit des comportements, des stratégies, bref crée de nouvelles réalités et en fait disparaître d'autres (Harrel-Bond, 1986). L'intérêt de travailler sur la vulnérabilité n'est donc pas tant de savoir qui est vraiment vulnérable, pauvre, à risque, fragile. II s'agit plutôt de retourner la question et d'étudier le discours lui-même ou comment les modèles sont construits discursivement, quels organismes et communautés académiques s'en nourrissent et les reproduisent. C'est l'ambition à laquelle participe cette étude.

Le corpus a été dépouillé manuellement à partir d'une série de mots-clés appartenant au champ lexical de la vulnérabilité et de leurs collocations. Outre le terme racine "vulnerab*", les termes "fragil*", "frail*", "danger" et les expressions "at risk", "risk* ", "in need of ", "need" ont été systématiquement recherchés et leurs collocations possibles (migrants, borderguards, foreigners, frontier, Frontex etc.) identifiées. Le label "victime" est étudié à part dans le corpus tant il est reconnu que l'emploi institutionnel de ce terme est moteur d'impuissance (Harrel-Bond, 1986; Thomas, 2010). Comme le souligne Hélène

16 L'article de Cambrezy fait partie d'un numéro spécial de la Revue Européenne des Migrations Internationales sur la vulnérabilité des migrants, $n^{\circ} 23$ (3) de 2007. Voir également le numéro spécial de la Revue des migrations Forcées (Forced Migration Review) intitulé "People Trafficking: upholding rights, understanding vulnerabilities", $\mathrm{n}^{\circ} 25$ de 2006. 
Thomas, décrire des personnes :

"Telles des victimes silencieuses du sort à partir de récits suscités par ceux qui les prennent en charge ou les étudient contribuent à les faire exister sous ces traits pitoyables." (Thomas, $2010: 34$ )

Le rôle de victime induit donc :

"Un traitement qui combine protection rapprochée et contrôle à distance' et fait des personnes " tantôt des martyrs médiatisés, tantôt des cibles discrètes d'interventions bienfaisantes, condamnées à la désubjectivation et au silence. " (Thomas, $2010: 2$ )

En lien avec la question des victimes se jouent également les termes "crime", "choice", "action". Pour finir, afin d'évaluer l'hypothèse de Campesi sur le discours humanitaire de Frontex, les termes "care" "protection" "rescue" "humanitarian" ont été retenus afin d'identifier les secouristes. Outre ces tournures, quelques éléments de structure grammaticale ainsi que des allégories et les métaphores ont été relevées, en particulier les images relatives à la naturalisation du risque (métaphores médicales, géographiques ou physiques). La recherche a été effectuée manuellement. En effet, l'étude n'avait pas pour objet d'établir une proximité statistique de termes clés, mais bien, pour chaque occurrence, de déterminer précisément qui est dit vulnérable, qui est dit à risque ou en danger. II s'agissait en outre d'identifier d'où viennent les risques. Or certains termes racines tels "risk" ou "danger" peuvent renvoyer tant aux sources qu'aux objets de menace. D'où l'intérêt d'une lecture humaine des textes plutôt qu'une revue automatisée des collocations. Par ailleurs la taille raisonnable du corpus des communiqués de presse le permettait. Pour les rapports, une variante simplifiée de la recherche a été effectuée.

\section{Les limites de l'enquête : interpréter avec prudence}

La valeur scientifique de l'enquête est bordée par les limites axiologiques de l'analyse discursive critique, par les limites du corpus, enfin par la méthode manuelle d'analyse. L'analyse discursive critique a été l'objet de nombreux débats en Science politique. On reconnaît aux chercheurs qui I'utilisent une capacité à prendre du recul par rapport à un objet d'étude, à questionner l'organisation, à capter des évolutions et des inflexions nouvelles, ou peu visibles. On leur reproche souvent un manque de rigueur dans le choix du corpus (parfois peu représentatif, voire biaisé) et une approche peu rigoureuse des collocations et concordances, notamment si la méthode ne fait pas état des garde-fous propres aux études statistiques (Baker et al., 2008). De fait, il peut être facile de trouver dans un texte les termes que I'on cherche. Enfin, lorsque la méthode est manuelle, elle peut être source d'erreurs techniques.

Dans notre cas, le corpus est représentatif et signifiant, une conséquence des principes de gouvernance et de transparence actuels. Par ailleurs, quelques jours suffisent pour télécharger et compiler les documents fondateurs, les archives complètes des rapports et communiqués de presse d'une organisation gouvernementale. Mais si ces contraintes de gouvernances rendent, a priori, les agences institutionnelles plus lisibles et plus redevables devant chercheurs et société civile, une contrepartie s'est imposée : la multiplication des textes, des images et des films mis en ligne fait obstacle à l'interprétation et diversion au 
sens. La masse agit contre le sens, d'autant qu'une novlangue chargée de jargon technique réécrit une réalité sociale et politique complexe. D'où l'importance de modes de décodage humains de telles masses textuelles qui permettent plus de finesse interprétative même s'ils sont coûteux en temps. Dans le cadre de cette recherche, l'étude des rapports de Frontex a dû être limitée à quelques termes clés. Quant aux risques d'erreurs manuelles, ils sont réels et ne peuvent être atténués que par une présentation des résultats qui en tienne compte. Dans cet article, seuls des écarts et variations importants seront considérés comme signifiants et présentés.

Autre limite, l'analyse sémantique est centrée sur le concept de vulnérabilité et donc des termes y afférant, choix qu'il est fort possible de critiquer. Comme indiqué ci-dessus, il s'agit dans cette étude de tester plusieurs affirmations et hypothèses sur Frontex circulant dans les mondes connexes universitaires et militants. Dans ce contexte cependant, il est important de reconnaître qu'aucune analyse textuelle, centrée principalement sur l'énoncé ne peut prétendre éclairer l'impact d'un discours institutionnel. En ce sens, dans la lignée du travail de Geert Jacobs sur le communiqué de presse, l'enquête présentée scrute l'autoprojection de Frontex dans l'espace public. L'impact d'un tel positionnement sur les journalistes, les partenaires ou encore les migrants relève d'une autre étude. De même, confronter le discours de Frontex à d'autres discours institutionnels (intertextualité) ou encore à la réalité des situations aux frontières de I'Europe devront être les sujets d'autres recherches, qui pourront s'appuyer sur les résultats ci-dessous.

\section{Des frontières et des hommes : qui est vulnérable?}

Quelques remarques préliminaires s'imposent : Frontex parle peu de vulnérabilité dans ses communiqués de presse, et jamais de fragilité. Les termes racines frail* et fragil* $^{*}$ sont totalement inexistants dans le corpus de communiqués de presse de l'agence. Lorsque la racine vulnerab* apparaît, c'est bien plus souvent en référence à des frontières et/ou des systèmes de contrôle de frontières, notamment les systèmes de contrôle aux frontières automatisés (Automated Border Control ou $A B C$ systèmes) qu'à des migrants. Les besoins dont s'inquiète I'agence sont autant les siens que ceux des autres (besoins financiers, opérationnels, en équipement, etc.). Par ailleurs, ces dernières années une rhétorique du risque et de son contrôle ont été façonnées et, depuis 2011, risque et vulnérabilité sont liés dans le cadre d'un modèle d'analyse du risque - CIRAM - que l'agence propose pour guider la prise de décision politique européenne.

\section{Des frontières sous pression}

"Doctors say that the best intensive care unit cannot replace prophylaxis. I would say that it applies to borders"

C'est avec cette métaphore médicale qu'lkka Laitinen, le directeur de Frontex exprimait dès 2007 ses craintes pour les frontières extérieures européennes et justifiait ses demandes d'augmentation de son budget. Ce discours, qui pose des frontières européennes vulnérables, n'a que peu évolué en dix ans. Sur le corpus de communiqués de presse, la racine "vulnérab* " apparaît une trentaine de fois dont un tiers en référence à des personnes (migrants, human beings, indi- 
viduals, group, trafficked persons and children) et deux tiers en référence aux frontières, aux systèmes de contrôle voire à la solidité des systèmes d'analyse mis en place (frontières, modèles d'évaluation, analyses, problèmes relatifs à l'automatisation des contrôles aux frontières, etc.). Ce n'est qu'en 2011 que le terme est mentionné en relation avec les frontières, usage qui se développe par la suite. Pour l'année 2015, année marquée par l'arrivée massive de réfugiés syriens en Europe, on ne trouve aucune trace du terme sur les 88 communiqués de presse de l'agence.

De même, le mot "victime" est relativement peu utilisé : moins de trentecinq occurrences dans une quinzaine de communiqués de presse qui annoncent des conférences ou des publications de rapports sur la traite humaine et seuls vingt-cinq des soixante-six rapports contiennent ce terme au moins une fois. De la même manière entre septembre 2015 et août 2016, ce terme n'a été employé que cinq fois dans les communiqués de presse, dont quatre en référence aux familles des victimes des attentats de Paris, Bruxelles et Nice, et une fois en référence à la traite humaine. De plus, il est principalement mentionné dans le cadre de l'expression consacrée "trafficking victims" ce qui met l'accent sur l'activité criminelle et donc relie immédiatement détresse et crime. Dans ce contexte l'agence se montre surtout intéressée par l'identification des victimes de la traite, y compris des victimes potentielles (notamment mineures) dont elle note le nombre croissant. Mis à part l'importance d'aider les douaniers à repérer ces personnes rapidement, peu de choses sont indiquées sur les victimes dans les communiqués de presse. Certains rapports sur les droits fondamentaux s'y attardent plus, mais ce n'est pas une question prégnante de l'ensemble des textes.

\section{Au-delà des expressions consacrées, quels besoins?}

La question du besoin est centrale à toute étude discursive de la vulnérabilité. Le corpus contient de nombreuses références à la notion (need/in need of). Néanmoins jusqu'en 2015, les manques auxquels Frontex fait référence sont presque uniquement les siens (besoins opérationnels, financiers, technologiques, humains, etc.), besoin de modèles d'analyse et besoins des modèles, besoins de I'UE, de ses États membres, nécessité de coordination, de coopération, de partage d'expérience, d'information, de fardeau, de soutien, etc. Ce sont donc bien les États membres de l'UE qui sont dans la nécessité; ce sont les systèmes techniques qui ont besoin de légitimité, de plus de développement et de recherche. L'agence Frontex, elle-même, se pose dans le besoin tout en se décrivant comme moteur de solutions et de plans d'action. L'expression "humanitarian need*" par exemple n'est utilisée qu'une seule fois en dix ans (2011). À partir de 2015 on rencontre une dizaine de fois l'expression «in need of protection" pour qualifier des migrants particulièrement vulnérables, une expression standardisée, quasiment jamais détaillée dans les communiqués de presse et peu dans les rapports associés ${ }^{17}$. En somme, les besoins des migrants ne dominent pas le discours de Frontex alors que ceux des institutions font l'objet de multiples publications, de conférences sur les avancées technologiques pour le contrôle de frontières, d'accords diplomatiques (notamment les

17 Excepté dans le détail d'une opération partage de peluches organisée par des agents Frontex scandinaves en juillet 2015. 
accords avec les pays tiers pour le partage d'information) et d'appel pour plus de moyens, d'engagement et même de solidarité. Le terme solidarité est employé une douzaine de fois en dix ans, toujours pour évoquer les relations entre États membres de I'UE, jamais en lien avec les migrants.

Dans ce même contexte, les questions de sauvetage sont relativement peu évoquées, et ce jusqu'en 2015. II est à noter que le concept de SAR (search and rescue) fait partie de listes d'activités par lesquelles Frontex tend à se définir (avec la surveillance, le contrôle aux frontières, etc.). Pourtant, jusqu'en 2015, seuls environ trois communiqués de presse par an usent du terme racine "rescu*" et encore moins du terme "sav*". 2015 par contre est l'année du sauvetage, à partir de février dans la Méditerranée centrale et ce jusqu'en septembre. Les termes racines "rescu* " et "sav* " s'interposent régulièrement dans la description des activités de l'agence sous différentes formes et notamment comme complément du nom (opérations de sauvetage, bateaux de sauvetage) ou épithète (surtout migrants secourus/sauvés) dans environ un tiers des communiqués de presse (vingt-neuf sur quatre-vingt-huit). Proportionnellement $c^{\prime}$ est nettement plus que les années précédentes et cela reflète la participation de l'agence à plusieurs grosses opérations de secours dans le cadre de l'opération Triton (suite à l'arrêt de l'opération de la marine italienne Marée Nostrum, d'ailleurs jamais mentionnée). Dans ce contexte, il est intéressant de noter que Frontex tente toujours de chiffrer sa participation, sa contribution au sauvetage. L'agence s'attribue environ un tiers des sauvetages et ne fait quasiment aucune allusion aux autres sauveteurs. Le travail de la marine italienne, des cargos privés, et des bateaux affrétés par des organisations humanitaires restent dans l'ombre, dans les silences de l'agence.

Paradoxalement, lors de ses opérations, Frontex offre secours à des personnes qui ne sont pas décrites comme en danger et dont les besoins ne sont pas la priorité de l'agence. L'organisation a aussi participé à la création de services intégrés d'accueil des migrants (création de hotspots en Grèce où s'organisent des interrogations de nouveaux migrants). Ses manuels indiquent d'ailleurs clairement que l'accès aux migrants et leur interrogatoire sont des dimensions clés de la collecte d'information (Frontex, 2012a : 33).

\section{Quelle rhétorique du risque?}

Contrairement à la sémantique de la vulnérabilité, la notion de risque elle sature les textes depuis les débuts des opérations en 2006. Différents rapports d'analyse de risques sont publiés à intervalles réguliers. Par risque, Frontex entend la probabilité de pénétration désordonnée encourue par chaque frontière extérieure de I'UE. À partir de 2011-2012, I'agence met en place un modèle intégré d'analyse de risques dont l'objet principal est de permettre une allocation optimale des moyens sur les frontières extérieures de l'union : le CIRAM. Ce modèle pose le risque comme résultante de trois composantes : la menace, la vulnérabilité et l'impact (Figure 1). 
Figure 1 : Le modèle d'analyse de risque CIRAM (Common Integrated Risk Analysis Model)

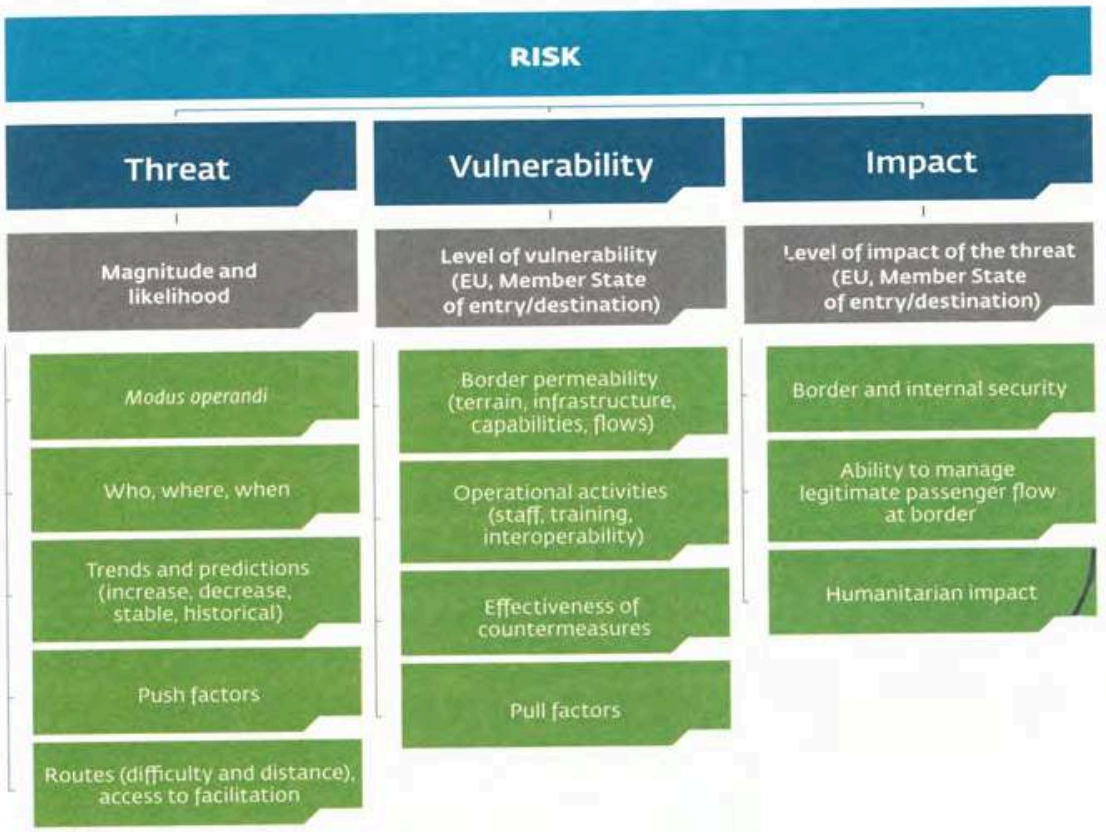

Source : Frontex Research and Analysis Unit, 2012.

L'analyse de la menace dans CIRAM met l'accent sur des phénomènes tels que l'évolution des réseaux de passeurs, l'état des routes terrestres ou maritimes menant à I'UE, ainsi que sur des facteurs de fuite, guerres, famines, etc. Le modèle construit donc la menace comme une donnée extérieure. Les facteurs de vulnérabilité des États membres sont la perméabilité physique de leurs frontières, leur incapacité à agir ou à mettre en œuvre des contre-mesures de surveillance et de contrôle ainsi que leur attractivité (sociale, économique, etc.). Si la menace vient de l'extérieur, la vulnérabilité, elle, est donc propre aux frontières et liée à leur ouverture sur le monde. Enfin l'impact se définit comme l'effet de la menace sur la sécurité dans I'UE et à ses frontières. L'impact, c'est donc le désordre aux frontières et les morts des migrants en Méditerranée font partie de la mesure de ce désordre.

Les trois variables du risque ne sont pas indépendantes ce qui rend le modèle complexe. II est à noter que les manuels d'explication de CIRAM vont jusqu'à présenter une fonction mathématique : du risque $R=f(T, V, I)$. Pourtant le lecteur est également averti des limites de ce que cette grille de lecture et ses chiffres peuvent signifier. II est même engagé ne pas se laisser abuser par un "faux sens" de la sécurité. L'importance d'évaluations qualitatives est ainsi soulignée. Étonnement, même dans les manuels d'utilisateurs, le diagramme reste peu explicite. La définition même de vulnérabilité donnée dans les rapports est incorrecte (ability to mitigate the threat - capacité à atténuer la menace). 
Comme tout modèle théorique, l'approche a ses fragilités scientifiques et certaines semblent reconnues par les analystes. C'est plutôt la présentation assurée de CIRAM par Frontex qui interroge. Nous sommes face à un schéma d'analyse pseudo mathématique par laquelle l'agence tente de s'imposer auprès de ses partenaires comme une organisation d'intelligence permettant de produire de l'ordre et de la sécurité. En témoigne le symbole qui marque ses rapports et surtout sa description sur les pages introductives des rapports (Figure 2).

Figure 2 : Le triangle, symbole des publications de Frontex

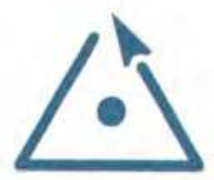

Source : Frontex, 2016.

"Formé par une flèche dessinant un triangle, avec un point au centre. Métaphoriquement, la flèche représente la nature cyclique du processus d'analyse de risque et son orientation vers des réponses opérationnelles appropriées. Le triangle est un symbole de proportions idéales et symbole de connaissance, reflétant la poursuite de l'exactitude factuelle, de la vérité et de l'analyse exhaustive. Le point au centre représente le facteur intelligence et le point focal où l'information de diverses sources converge pour être traitée, systématisée et partagée en produits analytiques."

(Frontex, 2012b : 2) $)^{18}$

Ce langage, qui oscille entre symbologie et scientisme, reste porteur d'un positionnement politique fort : on raconte un risque qu'on se dit en mesure d'évaluer. Pour ce faire, les rapports annuels (ARA) et trimestriels (FRAN) sur les frontières orientales et méridionales de I'UE détaillent des menaces en évolution constante et l'organisme annonce régulièrement de nouveaux partenariats comme clé d'une analyse dynamique et à la pointe:

"The heads of Frontex and Europol signed an agreement today to expand their cooperation in combatting cross-border criminal activities by exchanging information, including personal data of suspected criminals, and jointly planning operational activities.

"The new agreement represents a significant step forward in our cooperation, which is needed now more than ever as Europe grapples with various criminal activities linked to its external borders, along with the threat of terrorism," said Frontex Executive Director Fabrice Leggeri. ${ }^{19}$

"This week's meeting in Warsaw was the third of a series of workshops organised this year, the previous ones taking place in Ghana and Mauritania. As a result, Frontex produces annual reports, which analyse the irregular migratory movements affecting AFIC

18 Traduction de l'auteur.

19 Frontex, "Frontex and Europol agree to exchange information on cross-border crime", communiqué de presse, 04 décembre 2015. 
countries and EU Member States, cross-border criminality and provide an overview of the main regional security threats. ${ }^{20}$

On mentionne également une vulnérabilité dont on indique qu'elle est plus facile à mesurer que la menace, mais sur laquelle, finalement, on s'étend peu. La remarque suivante apparaît d'ailleurs dans plusieurs rapports :

"The risk assessment was done through examination of three main components of risk: threat, vulnerability and impact. However, vulnerabilities are not discussed in the present report meant for public release. " (Frontex, 2012c : 6)

La retenue est donc un choix. Peut-être a-t-il été jugé que donner l'information en ligne au grand public serait la donner aux passeurs. Les détails de la vulnérabilité seront sans doute transmis aux autorités compétentes, I'UE et ses États membres, vulnérables et donc prêts à allouer des ressources. En développant une rhétorique du risque adaptée aux peurs des gouvernants européens, en générant et distribuant de l'information de façon sélective, Frontex, petite agence, a réussi son pari de se positionner comme l'organisme qui sait où précisément se trouvent les maillons dits faibles de l'espace Schengen.

\section{La vulnérabilité contée par Frontex : retour sur la littérature et pistes de réflexion}

Notre court voyage au cœur des archives publiques de Frontex a mis en évidence que le risque et sa gestion priment, et de loin, sur l'attention à la vulnérabilité et que cette dernière concerne plus les États que des migrants. Ces résultats reposent sur les occurrences et les collocations émergeant de l'énoncé, ainsi que sur les absences et silences de l'énonciateur. Si cela peut nous amener à nuancer des accusations d'hypocrisie humanitaire souvent proférées à l'encontre de l'agence, cette étude soulève d'autres questions qui pourraient être approfondies par une recherche plus fine de l'intertextualité dans la construction des communiqués, et surtout des rapports (qui n'ont pu être exploités entièrement). Cette troisième partie propose donc quelques pistes de réflexion émergeant de l'étude de corpus. En particulier, elle suggère que la rhétorique du management du risque est une forme de fiction reposant sur une vision tronquée de la frontière et sur une confiance absolue en des solutions managériales et des remparts techniques.

\section{Quelle science-fiction?}

Frontex parle relativement peu de vulnérabilité, et moins de celle des migrants que de celle des frontières. L'agence parle avant tout d'elle-même, de son travail, de ses besoins. De fait, l'auto-référencement est permanent et le terme Frontex se retrouve plus de 1600 fois dans les archives des communiqués de presse (deux fois plus que celui de migrant lui-même très dominant). Avant toute chose, ce poids des mots suggère que l'organisme s'est projeté dans l'espace public comme l'intelligence seule capable de coordonner la protection des frontières extérieures européennes vulnérables aux risques de

20 Frontex, "Frontex hosts meeting of Africa-Frontex Intelligence Community (AFIC) ", communiqué de presse, 22 avril 2016. 
migration illégale. A contrario, rien dans notre étude ne permet d'affirmer que Frontex ait développé un discours humanitaire dominant. II semble plus juste de relever que l'agence choisit ses mots, ses silences et qu'un volet humanitaire se combine en période d'urgence (notamment en 2015) à la fonction première de l'agence : le filtre fin. Les publications et photo-reportages sur les sauvetages en mer de Frontex sont parfois mis en exergue par des lectures partielles de sa production écrite. Si un Forum sur les droits fondamentaux a bien été créé en 2011, son titre même indiquait qu'il n'était que consultatif.

Même l'iconographie de l'agence, pourtant un moyen puissant d'accentuer le pathos, restait en cohérence avec le discours centré sur le contrôle en 2016. Le bandeau qui ornait la page de garde du site internet les derniers mois de son existence officielle en est un bon exemple. Aucune des quatre photos en rotation permanente ne montrait de migrants, mais toutes représentaient des moyens techniques en mouvement $(4 \times 4$, hélicoptères) ou des gardes-frontières en action, scrutant I'horizon, s'entraidant, etc.

L'accent reste donc placé sur la technologie de surveillance et la solidarité douanière. De même, l'unique image de migrants ornant la page de garde introduisait un film sur le trafic humain et la façon dont les réseaux criminels de passeurs "profitent de la misère". Au-delà de la page d'accueil, on pouvait accéder au photo-reportage d'une opération SAR par laquelle un bateau de l'agence accoste une barque de migrants sur une mer d'huile et les embarque. Ces photos, si elles témoignent d'une dimension humanitaire réelle du travail des gardes-frontières, restent sobres. Seul trait surprenant de l'opération, les combinaisons de protection blanches des équipes de Frontex qui contrastent avec le dénuement des migrants secourus. Pourquoi ces casques et masques de protection du visage? Qui risque le plus dans l'opération? Finalement s'agit-il d'un sauvetage ou d'une arrestation? Ou des deux? Plutôt que de promouvoir une prétention humanitaire, les photos de Frontex illustrent le fait que le sauvetage, I'arraisonnement et l'accès au renseignement ne sont devenus qu'une seule opération, tout comme le migrant est à la fois un être humain, un passager illégal, une source d'information. Il peut même être vu comme un danger sanitaire.

Au-delà des pages de garde, les rapports de l'agence contiennent quelques photos de migrants, principalement de dos ou de loin, anonymes, souvent des groupes en marche. L'iconographie de ces rapports d'analyse reste néanmoins dominée par des graphiques, notamment des cartes pointant les flux entrant dans I'UE. Ces dernières, facilement reprises par les journalistes, mettent l'accent sur les maillons faibles à l'est et au sud de la chaîne frontière européenne.

\section{Crispation sur la frontière-ligne}

Si la charge d'hypocrisie ne peut être complètement retenue, I'histoire que nous conte Frontex intrigue néanmoins. À la question "Qui est vulnérable?" Frontex semble répondre : les États européens via leurs frontières extérieures. Cette affirmation, martelée pendant dix ans, a participé d'une vision tronquée des phénomènes migratoires et sécuritaires. Plus encore, ces représentations abordent la frontière comme une ligne géographique et n'envisagent, pour toute solution, qu'un recours à la technologie. En cela Frontex fausse la vision, enlisant ses lecteurs dans un syndrome digne de la Ligne Maginot. 
Les cartes de Frontex mettent I'accent sur la ligne et la flèche. La flèche donne une trajectoire, une direction, une percée. Son épaisseur indique la force du challenge, sa couleur (souvent rouge pour les flux non régionaux) alerte sur le danger, l'urgence ${ }^{21}$. Carte après carte, les flèches tournent et contournent les barrières européennes, comme à la recherche de points d'entrée moins surveillés. Pourtant, jusqu'en 2015, les passages en force du poste-frontière ou de la route sont rares. En mer, les harragas tentent leur chance de nuit, se font invisibles. La très grande majorité des passages ne se fait pas en masse ni même en flux, mais en gouttes d'eau individuelles qui à force de persévérance s'égrènent et se faufilent dans les interstices. Plus important encore, la grande majorité des entrées sur le territoire européen reste légale. De nombreuses personnes résidant irrégulièrement en Europe restent lorsque leur visa expire ou que leur demande d'asile est rejetée. Or le nombre d'entrées légales et son évolution n'apparaissent pas dans les cartes. De même les sorties de territoires sont rarement référencées. Les chiffres indiqués sont majoritairement des "détections" ou leur progression (pourcentages sur la carte donnée en exemple), la grande priorité de l'agence. Ces détections chiffrées à l'unité sans indication de marge d'erreur inquiètent et rassurent. Elles illustrent à la fois une pénétration et la capacité de Frontex d'en mesurer la force. La simplification est omniprésente. II manque aux histoires de Frontex, toute une mise en contexte. Les cartes, comme les textes, réduisent à des lignes et à des flèches des phénomènes migratoires pourtant complexes. Ces documents ignorent le fait que les espaces géographiques, les sphères de droit, les réseaux virtuels et les mouvements de personnes se superposent. Ils ignorent les communautés qui interagissent, de nouvelles formes de nomadisme, des trajectoires en zigzag ponctuées de pauses qui peuvent durer des années (Alioua, 2015) ${ }^{22}$.

L'étude conforte l'idée déjà avancée que l'agence promeut un mythe d'imperméabilisation des frontières (Peyronnet, 2015) et souligne une crispation remarquable du discours sur la frontière en tant que ligne. Si cette vision s'inscrit dans la lignée d'approches géopolitiques traditionnelles et sous-tend ce que Michel Foucher nomme la redécouverte des frontières pensées comme un "ensemble linéaire de points de franchissement" et de stratégies de contournement (Foucher, 2015), cette représentation reste néanmoins incomplète. Le travail de Frontex relève plus de la construction d'un filet très épais à multiples entrées et au maillage plus ou moins resserré que d'une ligne mur ou une barrière. La Méditerranée n'est pas un trait, mais un espace de circulation, de même que les pays de transit sont devenus des postes avancés de contrôle. Anthropologues, sociologues, historiens et géographes insistent sur l'importance d'aborder la frontière en termes d'espaces, parfois réduits à des sas, espaces que l'on peut même habiter (Agier, 2014 et 2016; Rinaudo, 2017). Le narratif frontésien lui s'inscrit dans la construction d'un imaginaire étroit et peu réflexif de la frontièreligne. De même pour la menace : cette dernière reste décrite comme une donnée extérieure alors que tout un pan des relations internationales explore depuis plus de vingt ans la construction politique des menaces au sein des études critiques de sécurité, et ce en particulier autour de l'objet «frontière» (Bigo, 2005

21 Pour une étude plus approfondie des enjeux de la représentation des phénomènes migratoires, voir Bacon et al. (2016).

22 Une complexité fort bien retracée par l'exposition «Frontières », Printemps 2016, Musée de I'Immigration, Paris. 
et 2011 ; Leonard, 2010; Huysmans, 2014). Chez Frontex, communiqués de presse comme rapports brillent par la répétition et l'auto-référencement. Négliger à ce point des apports universitaires importants sur les concepts de frontière et de menace relève d'une approche politique plus que scientifique. De fait, en dépit de son discours ${ }^{23}$, Frontex n'est pas un organisme scientifique, mais un acteur connecté à l'industrie plus qu'au monde universitaire. Cette industrie de la sécurité est d'ailleurs fortement sollicitée et encouragée dans la recherche de solutions techniques au filtrage sur la ligne. En cela, Frontex et ses partenaires nagent dans l'illusion du rempart technologique.

\section{La technologie comme seule option}

La lorgnette par laquelle Frontex envisage les frontières est donc étroite. Pour autant ses analystes restent conscients des limites des modèles utilisés et des précautions d'interprétation nécessaires.

"Increasing detection might be due to a rise in persons attempting illegal crossing or be a result of enhanced resources to detect such crossings. " (Frontex, $2011: 8$ )

Cette remarque technique illustre la question plus large de l'éclairage : si I'on braque les projecteurs sur nos frontières, si l'on accroît, comme on l'a fait ces dernières années, les ressources destinées à comptabiliser chaque passage, à retracer chaque trajectoire, alors on met en exergue des phénomènes dont on était peut-être peu conscients auparavant, mais dont on ne peut pas garantir qu'ils soient nouveaux. Les modèles d'analyse de Frontex sont trop récents, trop changeants, pour nous permettre le recul nécessaire à l'analyse des flux migratoires.

Que les modèles d'analyse aient leurs limites ne surprendra pas. Les difficultés commencent avec l'usage politique de rapports dits scientifiques et du pouvoir induit par ce rappel d'expertise. Si l'agence est réellement prise pour la source d'intelligence qu'elle prétend être, alors ses appels comme ses silences génèrent des impacts. Hurler à la crise par exemple est un acte politique par lequel on déclare le danger imminent et on requiert une mobilisation exceptionnelle. En 2010 Frontex reportait une augmentation de la proportion du nombre de mineurs (notamment d'Afghanistan) demandant I'asile en Europe ${ }^{24}$. Aucune crise pourtant n'a été déclarée et la question des mineurs isolés n'a fait qu'empirer au point qu'en 2015, l'UE aurait perdu la trace de près de 10000 mineurs entrés sur ses territoires ${ }^{25}$. Le silence de l'agence sur la disparition d'adolescents (aucune réaction aux déclarations d'Europol), couvert en 2016 par plusieurs appels à conférence sur les technologies de surveillance illustre une orientation

\footnotetext{
23 Par exemple, concernant la mise en œuvre de standards de formation pour les douaniers "During the development process up to the date and scientific principles were followed", communiqué de presse, 04 mars 2008; ou encore "From Frontex side, experts with scientific, technical and operational background will attend", communiqué de presse, 25 mars 2014.

24 Frontex, "Frontex Releases Report on Unaccompanied Minors in European Migration ", communiqué de presse, 13 décembre 2010.

25 The Guardian (2016) 10,000 refugee children are missing says Europol, [en ligne], consulté le 03/11/2016. URL : https://www.theguardian.com/world/2016/jan/30/fears-formissing-child-refugees. EUROPOL avait souligné le problème dès I'automne 2015, puis confirmé l'estimation de 10000 jeunes non traçables en janvier 2016.
} 
obstinée et une quête remarquable de solutions techniques, un choix éminemment politique. Au cours des dix dernières années, Frontex s'est fait grand promoteur de la technologie de détection (frégates, satellites, drones, biométrie, etc.). Cette infrastructure est pourtant coûteuse, son impact sur les libertés civiles non négligeables (Thomas, 2006; Loukinas, 2017), enfin sa fiabilité questionnable aux vues des événements de ces dernières années.

Alors que les outils technologiques sont invités au secours de frontièreslignes malmenées, l'outil juridique lui se fossilise. Bien que le droit soit censé encadrer les pratiques, les références aux textes et termes légaux sont relativement rares dans la communication de Frontex. De plus, ce sont principalement des expressions consacrées : "fondamental rights" "trafficking victims", expressions finalement "trop sacrées" pour être signifiantes ou engageantes. Aucune conférence sur les droits des personnes n'est signalée dans les conférences de presse. Le dernier rapport du Comité Consultatif sur les Droits Fondamentaux (créé en 2011) met en évidence le manque de moyens et d'accès donné aux membres du comité. Cette marginalisation de l'outil juridique et des institutions y afférant contribue à occulter des pans entiers de la réalité du travail de Frontex et notamment le coût humain des politiques d'étanchéité sur la ligne. II est possible que l'exclusion du droit soit le produit naturel de la vision amétrope de l'agence dont le travail est apparemment centré sur la bataille sur la ligne, alors que le droit lui gère les relations humaines et institutionnelles sur des territoires, des espaces. Il est également possible que la marginalisation de I'outil juridique soit une conséquence malheureuse du manque d'anticipation et de la gestion en mode urgence-crise. De fait, Frontex se construit dans la réaction, insiste toujours sur l'urgence et le besoin. L'agence se positionne donc dans la crise, cette contraction de l'espace et du temps qui induit souvent des décisions abruptes et, implicitement, une exclusion du droit. II reste néanmoins à déterminer si ce positionnement ne relève pas, tout simplement, d'un choix politique explicite.

\section{Conclusion : les risques de la science-fiction}

Nombre de travaux insistent sur l'idée que Frontex nous raconte une histoire qu'il faut regarder de près. Cette étude suggère que Frontex défend une vision particulière de la situation aux frontières : un scénario fait de "risques" et de «crimes» menaçant les États membres européens, scénario dans lequel la sémantique de la vulnérabilité des personnes figure relativement peu. En ce sens l'agence reste jusqu'en 2016, "l'agence tout risque" décrite par Rodier (2010). Frontex, omniprésent, se pose en acteur clé de certains sauvetages, mais surtout en expert de l'analyse. L'agence se veut incontournable moins dans I'action aux frontières que dans sa coordination, notamment aux moments de crises, ces contractions de l'espace-temps qui produisent souvent courts-circuits et confusions. L'étude suggère aussi que l'accent mis sur la ligne sans penser I'espace et sur la technologie à l'exclusion du droit produit une vision myope de la question des migrations aux frontières européennes.

Pendant dix ans, Frontex s'est concentré sur l'analyse d'un risque fait de menaces extérieures et de vulnérabilités intérieures, sur l'anticipation de ce risque, sur la construction de modèles de risques. Tout cela au risque de l'engen- 
drer. Non seulement les politiques de blocage des flux (légaux et physiques) aux frontières et dans des pays tiers ont conduit à des effets pervers en matière de criminalité, de violence, de trafic, et de morts, mais la rhétorique du risque a conforté l'image d'une Europe assiégée et dans la réaction face à une menace extérieure grandissante. Aux vues des positions des gouvernements européens depuis 2015, la question de la performativité d'un tel discours sécuritaire se pose. II reste donc à évaluer précisément dans quelle mesure le discours narratif de Frontex a contribué à la construction de visions et de politiques étroites, voire contre-productives. Ce qui est établi néanmoins, c'est que pour s'imposer comme référence sur la sécurité des frontières auprès des États membres, la petite agence européenne a crié au feu pendant dix ans et, somme toute, a joué au pompier pyromane.

\section{Références bibliographiques}

Agier Michel (2016) Nouvelles réflexions sur les lieux des sans-États, Multitudes, $64(3)$, pp. 53-61.

Agier Michel (2014) Un monde de camps, Paris, La Découverte, 350 p.

Alioua Mhedi (2015) Vivre sur la route en attendant de passer la frontière, in Yvan Gastaut et Catherine Wihtol de Wenden Éds., Frontières, Paris, Magellan et Cie, pp. 100-105.

Amnesty International (2013) Frontier Europe, Human Rights abuses on Greece's borders with Turkey, Rapport, 40 ps.

Bacon Lucie, Clochard Olivier, Honoré Thomas, Lambert Nicolas, Mekdjian Sarah et Rekacewicz Philippe (2016) Cartographier les mouvements migratoires, Revue Européenne des Migrations Internationales, 32 (3-4), [en ligne] consulté le 12/09/2017. URL : http://remi.revues.org/8249

Baker Paul, Gabrielatos Costas, Khosravinik Majid, Krzyzanowski Michal, McEnery Tony, and Wodak Ruth (2008) A useful methodological synergy? Combining Critical Discourse Analysis and Corpus Linguistics to examine discourses of Refugees and Asylum Seekers in the UK Press, Discourse and Society, 19, pp. 273-306.

Bauloz Céline (2017) The EU Migration Partnership Framework: an external solution to the crisis?, EU Immigration and Asylum Law and Policy, Blogpost, Odysseus Academic Network, [online] URL: http://eumigrationlawblog.eu/the-eumigration-partnership-framework-an-external-solution-to-the-crisis/

Beauchemin Chris et Lessault David (2014) Les statistiques des migrations africaines: ni exode, ni invasion, e-Migrinter, 12, pp. 32-43, [en ligne] consulté le 05/02/2017. URL : https://e-migrinter.revues.org/417

Bigo Didier (2011) Le "Nexus", sécurité, frontière et migration : programme et diagramme, Culture et Conflits, 84, pp. 7-12.

Bigo Didier (2005) La mondialisation de I'(in)sécurité?, Culture et Conflits, 58, pp. 53-101.

Bresson Maryse, Geronimi Vincent et Pottier Nathalie (Dirs.) (2013) La vulnérabilité : questions de recherche en sciences sociales, Fribourg, Academic Press Fribourg, 299 p. 
Cambrezy Luc (2007) Réfugiés et migrants en Afrique : quel statut pour quelle vulnérabilité?, Revue Européenne des Migrations Internationales, 23 (3), pp. 13-28.

Cambrezy Luc et CharbitYves (2007) Éditorial, Revue Européenne des Migrations Internationales, 23 (3), pp. 7-11.

Campbell Zach (2016) Shoot First: coast guard fired at Migrant Boats, European Border Agency Documents Show, The Intercept, 22/08/2016, [online] last checked on 01/02/2017. URL : https://theintercept.com/2016/08/22/coast-guard-fired-at-migrant-boats-european-border-agency-documents-show/

Campesi Guiseppe (2014) Frontex, the Euro-Mediterranean border and the paradoxes of humanitarian rhetoric, South East European Journal of Political Science, 11 (3), pp. 126-134.

Fairclough Norman (2003) Analysing Discours, Textual Research for Social Research, London, Routledge, 288 p.

Fairclough Norman (1995) Critical Discourse Analysis, the Critical Study of Language, London, Longman, 265 p.

Fink Melanie (2012) Frontex Working Arrangements: Legitimacy and Human Rights Concerns Regarding "Technical Relationships", Merlourios Utrecht Journal of International and European Law, 28 (75), pp. 20-35.

Foucher Michel (2015) La réaffirmation des frontières, in Yvan Gastaut et Catherine Wihtol de Wenden Éds., Frontières, Paris, Magellan et Cie, pp. 30-35.

Frontex (2011) Eastern Borders Risk Analysis Network. Annual Overview 2011, $34 \mathrm{p}$.

Frontex (2012a) Guidelines for Risk Analysis Units, Structure and tools for the application of CIRAM, version 2.0, 112 pages.

Frontex (2012b) Common Integrated Risk Analysis Model, a comprehensive update, version 2.0, $52 \mathrm{p}$.

Frontex (2012c) Western Balkans. Annual Risk Analysis 2012, 48 p.

Harrel-Bond Barbara (1986) Imposing Aid, Emergency Assistance to Refugees, Oxford, Oxford University Press, 440 p.

Horsti Karina (2012) Humanitarian discourse legitimating migration control: Frontex public communication, in Michi Messer, Renee Schroeder and Ruth Wodak Eds., Migrations: Interdisciplinary Perspectives, pp. 297-308.

Huysmans Jeff (2014) Security Unbound, Enacting Democratic Limits, Oxon, Routledge, 226 p.

Intran Caroline et Sibley Anna (2014) Faire sombrer Frontex, Plein Droit, 103 (4), pp. 40-43.

Jacobs Geert (1998) Projected Discourse: An Analysis of Receiver Roles in Press Releases, Text: Interdisciplinary Journal for the Study of Discourse, 18 (4), pp. 505-523.

Leonard Sarah (2010) EU Border security and migration into the EU: Frontex and securisation through practices, Journal of European Security, 19 (2), pp. 231-254. 
Loukinas Panagiotis (2017) Surveillance and Drones at Greek Borderzones: Challenging Human Rights and Democracy, Surveillance \& Society, 15 (3-4), pp. 439-446, [online]last checked one 11/09/2017. URL : https://ojs.library.queensu. ca/index.php/surveillance-and-society/article/view/6613

Ottavy Eva et Clochard Olivier (2014) Franchir les dispositifs établis par Frontex. Coopérations policières transfrontalières et refoulements en mer Egée, Revue Européenne des Migrations Internationales, 30 (2), pp. 137-156.

Peyronnet Chloé (2015) Le programme de travail de Frontex pour 2015 : une fuite en avant mal dissimulée, La Revue des droits de l'homme, [en ligne] consulté le 24/06/2016. URL : https://revdh.revues.org/1373

Rinaudo Christian (2017) El Norte. Une sociologie de la frontière et des dynamiques migratoires, Lectures Anthropologiques, 3, [en ligne] consulté le 05/02/2018. URL : http://92.222.82.244/lodel/lecturesanthropologiques/index. php?id=443

Rodier Claire (2010) Frontex, I'agence tout risque, Plein Droit, 87 (4), pp. 8-11.

Schneider Florian (2013) How to do Discours Analysis, A Tool Box for Analysing Political Tests, [online] last checked on 29/08/2016. URL : www.politicseastasia. com/studying/how-to-do-discourse-analysis/

Sleurs Kim, Jacobs Geert and Van Waes Luuk (2003) Constructing Press Releases, Constructing Quotations: A Case Study, Journal of Sociolinguistics, 7 (2), pp. 102-212.

Thomas Hélène (2010) Les vulnérables, la démocratie contre les pauvres, Bellecombe en Bauges, Le Croquant, [en ligne] consulté le 29/04/2016. URL : http://www.reseau-terra.eu/IMG/pdf/LV.pdf

Thomas Hélène (2008) Vulnérabilité, fragilité, précarité, résilience, etc., Recueil Alexandries, Collections Esquisses, [en ligne] consulté le 27/06/2016. URL : http:// www.reseau-terra.eu/article697.html

Thomas Rebekah (2006) Biometrics, International Migrants and Human Rights, European Journal of Migration and Law, 7 (4), pp. 377-411.

United Nations (2013) Report of the Special Rapporteur on the human rights of migrants, A/HRC/23/46/Add.4, $22 \mathrm{p}$.

Van Dijk Teun A. (2009) Texte, Contexte et Connaissance, Semen, 27, [en ligne] consulté le 05/11/2017. URL : http://semen.revues.org/8890 


\section{Cécile Dubernet}

\section{Qui est vulnérable ? Une analyse critique du discours public de l'agence européenne Frontex}

Cet article présente les résultats d'une analyse critique des communiqués de presse et rapports de l'agence européenne Frontex sur les dix années de sa période opératoire (2006-2016). L'étude du vocabulaire dominant, des thèmes récurrents et des jeux de langage met en évidence la permanence d'une rhétorique du risque soulignant des menaces extérieures aux frontières de I'UE et des vulnérabilités propres. Malgré quelques réflexions sur les droits fondamentaux ou le sauvetage en mer, la vulnérabilité dont parle l'agence reste celle des États européens, non des migrants. Par ailleurs, l'agence se présente comme l'intelligence seule capable d'orienter et de coordonner la sécurisation des frontières. L'attention portée aux mots de l'archive sur la durée permet de nuancer les accusations d'hypocrisie humanitaire régulièrement lancées à l'encontre de Frontex. Ce travail permet également de souligner la structure d'un discours. On note en particulier une crispation sur la frontière en tant que ligne, sur la menace vue comme extérieure et sur la technologie construite comme solution. Compte tenu de la mue de Frontex en 2016, cette étude ouvre la voie à des analyses plus poussées tant sur la marginalisation de la protection juridique des migrants que sur la performativité d'un narratif institutionnel.

\section{Who Is Vulnerable? A Critical Analysis of the Public Narrative of the European Agency Frontex}

This article presents the results of a critical analysis of the press releases and reports of the European agency Frontex over its ten years of operational activities (2006-2016). The study of dominant vocabulary, recurrent themes and language interplays emphasises the permanence of a rhetoric of risk, emphasizing external threats at the borders of the EU and internal vulnerabilities. Despite some references to Fundamental Rights or to search and rescue operations, the vulnerability at the core of the narrative remains that of EU members, not that of migrants. In addition, the agency keeps positioning itself as the intelligence organisation best able to shape and to coordinate the protection of borders. Indeed, paying attention to the words of the archives helps to nuance our judgement on the hypocrisy of Frontex. This work also highlights the structure of a discourse, in particular its focus on borders seen as "lines", on threats seen as "external" and on technology seen as "solution". Given the transformation of Frontex in the course of 2016, this study calls for further critical enquiries not only on the marginalisation of migrant juridical protection but also on the performativity of an institutional narrative. 


\section{¿Quién es vulnerable? Un análisis crítico del discurso público de la agencia europea Frontex}

Este artículo presenta los resultados de un análisis crítico de comunicados de prensa e informes de la agencia europea Frontex sobre sus diez años de actividad (2006-2016). El estudio del vocabulario dominante, de los temas recurrentes y de los juegos de palabra revela la continuidad de una retórica del riesgo que pone en evidencia las amenazas exteriores a las fronteras de la UE y su vulnerabilidad. El concepto de vulnerabilidad es utilizado por la agencia a veces en relación a los derechos fundamentales y al rescate marítimo de los migrantes, pero sobretodo en relación a las fronteras. Por otro lado, Frontex se presenta como la única organización capaz de asegurar las fronteras El trabajo realizado sobre el lenguaje durante un periodo de diez años, permite relativizar la acusación de hipocresía dirigida regularmente contra Frontex. Este trabajo ayuda igualmente a poner en evidencia un discurso estructurado en torno a tres ideas principales: la frontera como línea, la amenaza como exterior, y la tecnología como solución. Dada la transformación de Frontex en 2016, este estudio abre la reflexión sobre la marginalización de la protección jurídica de los migrantes y del impacto de una narración institucional. 